\title{
«Wir müssen die Vorteile des EPD aufzeigen»
}

\section{Interview: Bruno Kesseli}

Dr. med. et lic. phil., Chefredaktor

Seit 15. April dieses Jahres ist das Bundesgesetz über das Elektronische Patientendossier (EPD) in Kraft. Seither ist es ziemlich ruhig geworden um dieses Grossprojekt, das die Behandlung der Patienten verbessern und effizienter machen soll. Im Gespräch gibt Pascal Strupler Auskunft über den Stand der Umsetzung. Der Direktor des Bundesamts für Gesundheit (BAG) zeigt sich von den Vorteilen des EPD überzeugt und nimmt auch Stellung zu möglichen Stolpersteinen.

Herr Strupler, wenn man das Projekt «Elektronisches Patientendossier» (EPD) als Marathonlauf ansieht: Wo stehen wir heute?

Der Marathon begann für mich im Mai 2013 mit der Verabschiedung der Botschaft des Bundesrates zum Bundesgesetz über das Elektronische Patientendossier an das Parlament. Die Zeit vorher hatte eher noch den

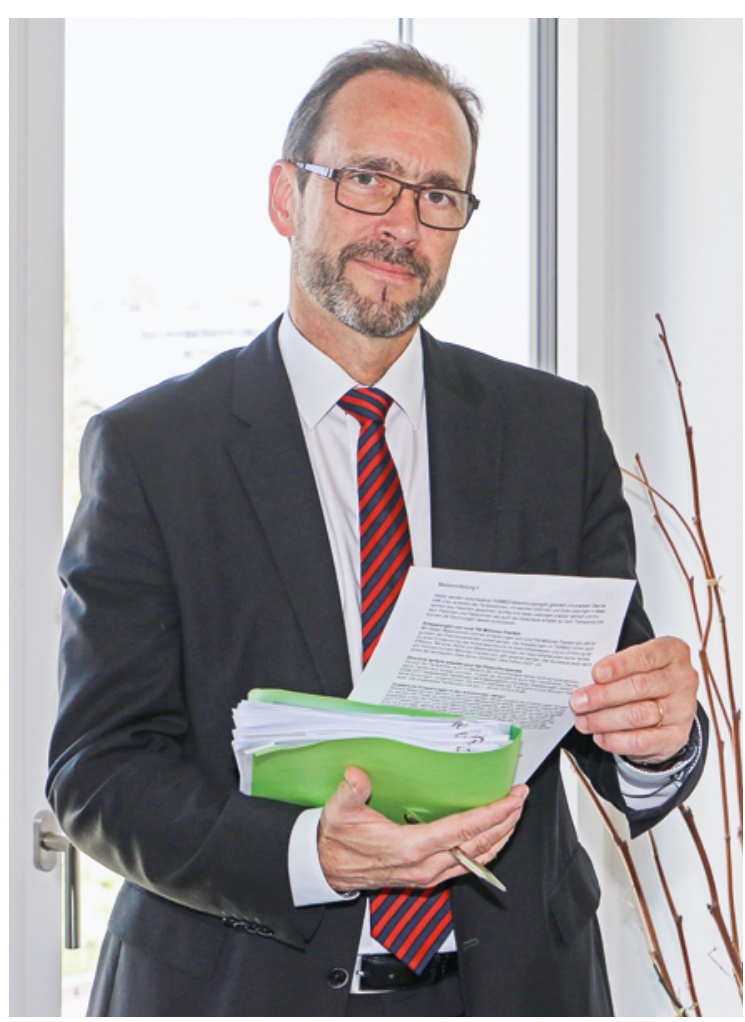

"Was nun folgt, ist Knochenarbeit»: BAG-Direktor Pascal Strupler zur Einführung des EPD
Charakter eines Trainingslagers. Das Koordinationsorgan zwischen Bund und Kantonen eHealth Suisse war ja schon 2007 gegründet worden. Eine Grundlagenarbeit war bereits geleistet und mögliche Modelle waren geprüft worden. Seit April 2017 sind nun Gesetz und Verordnungen in Kraft, aber wir haben natürlich noch kein EPD. Ich würde sagen, drei Viertel des Marathons sind absolviert, aber was nun folgt, ist Knochenarbeit.

\section{Worin besteht diese primär?}

Es wird viel Überzeugungsarbeit brauchen, viele Informationen müssen an verschiedenen Orten ankommen. Zudem müssen noch viele technische und organisatorische Detailfragen geklärt werden. Und bei alledem darf man das Ziel nicht aus den Augen verlieren.

Sie und diverse Spezialisten beschäftigen sich schon seit Jahren mit dem EPD. Wenn man sich dagegen in der Ärzteschaft und bei den Patienten umhört, hat man nicht den Eindruck, dass das Thema dort schon richtig angekommen ist.

Ja, diesen Eindruck teile ich. Wir haben deshalb mit eHealth Suisse und den Kantonen eine Art Fahrplan mit Aufgaben erstellt, die bis zur Einführung des EPD noch zu erledigen sind. Ein wesentlicher Teil der Arbeit wird darin bestehen, zu informieren. Dabei stehen die Ärzte und die Ärztegesellschaften ganz oben auf der Liste.

Die Ärzteschaft ist allerdings sehr heterogen.

Wir haben zwar die Gesamtheit der Ärztinnen und Ärzte im Auge, aber die Voraussetzungen sind tatsäch- 
lich sehr unterschiedlich. Ein Teil der älteren Generation wird sich wohl nicht mehr auf das EPD einlassen wollen. Dies ist auch nachvollziehbar, wenn jemand weiss, dass er oder sie in fünf Jahren in Pension geht. Ein gewisser Anreiz könnte aber darin be-

«Ein Teil der älteren Generation wird sich wohl nicht mehr auf das EPD einlassen wollen. Dies ist auch nachvollziehbar.»

stehen, dass man die Praxis technisch auf dem aktuellen Stand übergeben möchte. Dazu würde auch gehören, dass die Patienten über ein EPD verfügen. Diese Informationsarbeit hat in nächster Zeit hohe Priorität.

Wie sieht es in Bezug auf die Patienten aus? Täuscht der Eindruck, dass erst eine verschwindende Minderheit über das EPD Bescheid weiss?

Wir haben diesbezüglich ein sequenzielles Vorgehen geplant. Zuerst müssen die Ärztinnen und Ärzte über das EPD im Bild sein, weil sie die ersten Ansprechpartner der Patienten sein werden. Es ist aber völlig klar, dass die Patienten auch direkt angesprochen und informiert werden müssen. Auf der Website www.patientendossier.ch werden die grundlegenden Informationen zum EPD in gut verständlicher Form vermittelt, auch mit kurzen Videofilmen. Daneben gibt es auch eine Broschüre, die demselben Zweck dient. Die Informationen für Patienten werden in den nächsten Monaten und nochmals Anfang 2018 intensiviert.

Bleiben wir noch etwas beim Bild des Marathons. Wie sieht die Rolle des Bundes in diesem Rennen aus? Ist er der Pacemaker, der irgendwann aussteigt, der Organisator, der Rahmenbedingungen definiert und für geordnete Abläufe sorgt, oder hat er mehrere Rollen inne ...?

Der Bund und die Kantone sind die Organisatoren. Da es sich um ein Langstreckenrennen handelt, ist der Organisationsaufwand beträchtlich. Eine wichtige Aufgabe besteht darin, das Rennen so attraktiv zu gestalten, dass die Ansprechpartner mitmachen. Dazu gehört beispielsweise der finanzielle Anreiz, den der Bund durch die Unterstützung der Gemeinschaften und Stammgemeinschaften geschaffen hat.

Für die Stammgemeinschaften gibt es finanzielle Unterstützung, nicht aber für die einzelnen Praxen. Weshalb wurde so entschieden?

Es ist richtig, dass dies in den Kommissionen und im Plenum diskutiert und so entschieden wurde. Es han- delt sich um einen politischen Entscheid, der so hinzunehmen ist.

\section{Schrittmacher ist der Bund teilweise auch. Spitäler beispielsweise haben die verbindliche Vorgabe, das EPD innerhalb von drei Jahren einzuführen.}

Ja, der Gesetzgeber hat in der Tat für Spitäler und Heime diesen Zeitrahmen vorgegeben. Ansonsten gilt aber die doppelte Freiwilligkeit: Sowohl für die Ärzte wie auch für die Patienten ist das Eröffnen beziehungsweise Führen eines EPD freiwillig.

\section{Wie wollen Sie in der Ärzteschaft die Motivation} fördern, beim EPD mitzumachen?

Das Hauptinstrument ist aus meiner Sicht die Information. Wir müssen die Vorteile des EPD für den Patienten, aber mittel- und langfristig auch für die Ärztinnen und Ärzte aufzeigen. Dazu sollten auch Hilfestellungen bei Anlaufschwierigkeiten geboten werden, beispielsweise Open-Source-Anbindungslösungen an das EPD für die Praxis und Klinikinformationssysteme.

«Eine wichtige Aufgabe besteht darin, das Rennen so attraktiv zu gestalten, dass die Ansprechpartner mitmachen.»

Werden wir nun etwas konkreter. Welche Verbesserungen der in der Schweiz bereits sehr guten Gesundheitsversorgung erhofft man sich von der Einführung des EPD?

Die Verbesserungen für die Gesundheitsfachpersonen liegen primär darin, dass auf das Dossier unabhängig von Zeit und Ort zugegriffen werden kann. Konkret wird die Informationsübertragung beschleunigt, was beispielsweise Doppeluntersuchungen zu vermeiden hilft. Die Patienten werden von einer gewissen «Briefträgerfunktion» entlastet, Röntgenbilder, Kurzaustrittsberichte oder Rezepte müssen sie dann nicht mehr herumtragen. Dies alles findet sich dann auf dem EPD. Auch die Patientensicherheit wird verbessert, indem beispielsweise sofort ersichtlich ist, welche Medikamente ein Patient einnimmt oder an welchen Allergien er leidet. Gerade bei polymorbiden Patienten, die von mehreren Ärzten behandelt werden, ist nicht immer gewährleistet, dass jeder weiss, was der andere verordnet hat.

Zumindest in der Theorie sieht das durchaus überzeugend aus. Sehen Sie auch Problemzonen des Konzepts EPD?

Die Ärztinnen und Ärzte müssen bereit sein, überhaupt in das Projekt einzusteigen. Dazu braucht es 
eine gewisse Infrastruktur. Weiter muss es gelingen, die Patienten gut über das EPD zu informieren, insbesondere auch über Aspekte der Datensicherheit. Das "Vertrauen ins System» ist für mich eigentlich der zentrale Aspekt. Alle Beteiligten, insbesondere natürlich die Patienten, müssen die Gewissheit haben, dass die im EPD abgelegten Daten nicht missbräuchlich verwendet werden. Und sie werden die Kontrolle darüber haben, wer auf die abgelegten Daten zugreifen kann.

\section{«Das 'Vertrauen ins System' ist für mich eigentlich der zentrale Aspekt.»}

Die Probleme liegen aus Ihrer Sicht also nicht in der Sache selbst, sondern auf der Motivationsebene? Wie gesagt, es braucht beides: die Bereitschaft und die Infrastruktur.

Aus den Reihen verschiedener Interessengruppen war zeitweise die Kritik zu hören, das Projekt sei sehr stark top-down entwickelt worden.

Ich habe zwar davon gehört, dass es bei der Ausarbeitung der Verordnungen Diskussionen gegeben hat. Nach meiner Einschätzung konnten die Probleme aber gelöst und die relevanten Akteure eingebunden werden. Ich würde sogar so weit gehen und sagen, dass die Zusammenarbeit aller Beteiligten beim EPD besonders ausgeprägt war.

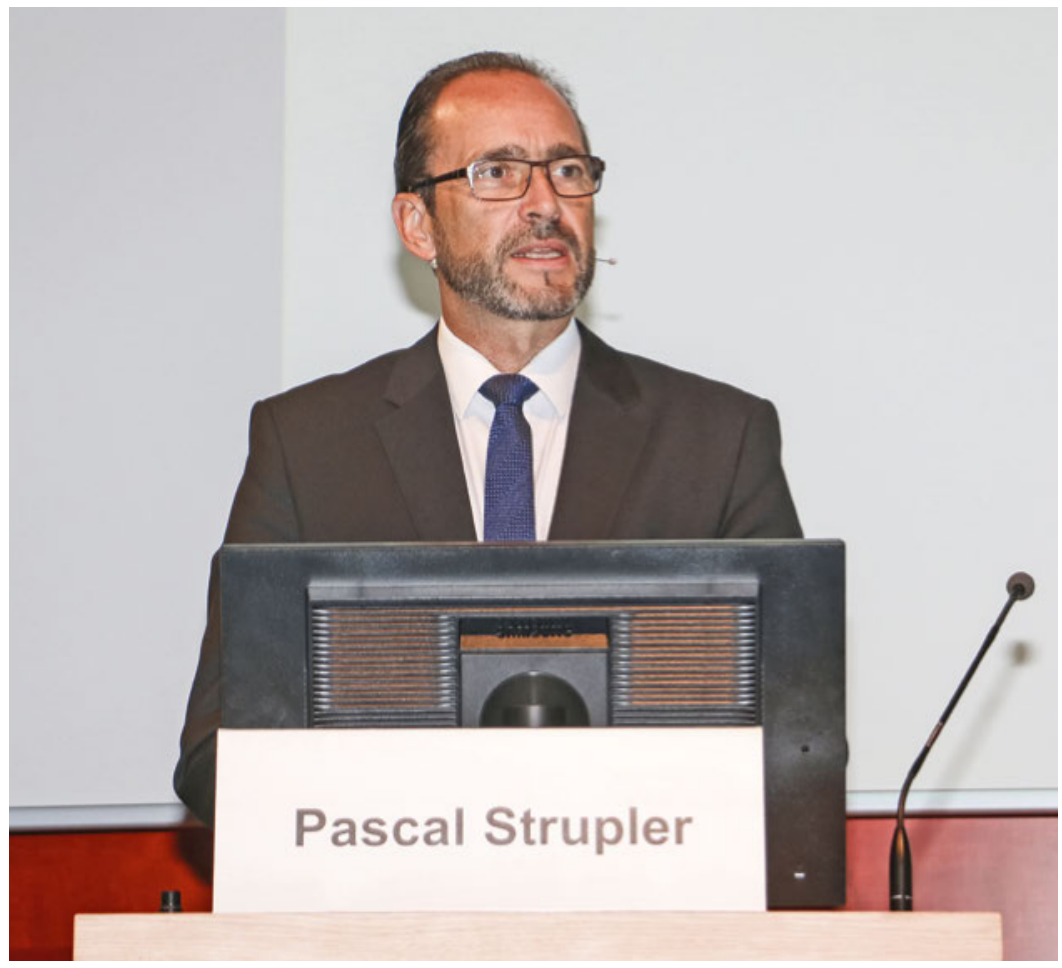

Pascal Strupler ist überzeugt, dass das EPD die Behandlung der Patientinnen und Patienten verbessern wird.
Ein Ziel, das im Zusammenhang mit dem EPD immer wieder genannt wird, ist die Stärkung der Gesundheitskompetenz der Patienten. Inwiefern trägt ein EPD zu einem solchen Ziel bei?

Zunächst müsste man sich auf eine Definition von $\mathrm{Ge}$ sundheitskompetenz einigen. Dazu gehört, sich Informationen zu Gesundheitsfragen zu beschaffen, diese $\mathrm{zu}$ verstehen und daraus Entscheide in Bezug auf die eigene Gesundheit ableiten zu können. Aufgrund der vielen Informationen, die heute auf verschiedenen Kanälen zur Verfügung stehen, wissen Patienten immer besser über ihren Gesundheitszustand, über ihre Krankheiten Bescheid. Der Vorteil des EPD ist, dass es die behandlungsrelevanten Informationen enthält. Da der Patient Einblick in das Dossier hat, kann er die wichtigen medizinischen Schritte nachvollziehen. Er kann aber auch eigene Messdaten ins Dossier einspeisen, was je länger je mehr von Bedeutung sein wird. All dies wird dazu führen, dass Ärzte und Patienten besser auf die Sprechstunde vorbereitet sein werden, dass dort die wichtigen Fragen gezielt angesprochen werden.

\section{Das wirft aber Fragen auf zur Qualität und Menge der} auf dem EPD gespeicherten Daten. Wer ist dafür besorgt, dass die abgelegten Daten tatsächlich relevant und aktuell sind?

Dafür gibt es keinen Hauptverantwortlichen. Wer als Patient selbst Einträge vornimmt, ist Herr seiner Daten und selbst verantwortlich für die abgelegten Inhalte. Bei den Ärzten gehen wir davon aus, dass die Einträge korrekt sind, und die Ärztinnen und Ärzte können die gemachten Einträge selbstverständlich mit den Patienten diskutieren. Obsolete oder nicht mehr relevante Informationen können in Absprache gelöscht werden.

\section{Dass potenziell sehr viele Akteure ins EPD involviert sind, sehen Sie nicht als Problem? Polymorbide Patienten werden häufig von verschiedenen Ärzten, Therapeuten und Institutionen betreut.}

Ich nehme das EPD nicht als Problem, sondern als die Lösung wahr. Denn die von Ihnen beschriebene Realität gibt es. Das EPD ermöglicht es eben gerade, die Übersicht zu haben.

\section{Der Zeitaufwand für die Pflege des EPD dürfte} beträchtlich sein, insbesondere in der Hausarztpraxis, die am Schluss wahrscheinlich doch die zentrale Verwaltungsstelle sein wird. Wie werden solche Leistungen abgegolten? Wäre dies ein Fall für die Rubrik "Ärztliche Leistung in Abwesenheit des Patienten"?

Bereits heute werden Arbeiten an der Krankengeschichte des Patienten abgegolten. 
Vielen Ärztinnen und Ärzten fehlt vor allem eines: Zeit für ihre Patientinnen und Patienten. Wenn nun neben der Krankengeschichte pro Praxis noch Tausende von EPD à jour gehalten werden müssen, dürfte das die Situation nicht verbessern ...

Bei jeder Umstellung, bei jeder neuen Technologie, die ins Berufsleben integriert werden muss, gibt es eine Anlaufphase, in der zusätzlich Zeit investiert werden muss. Das soll nicht abgestritten werden, kommt aber in jedem Berufsfeld vor und darf erwartet werden. Man sollte Aufwand und Nutzen im Zusammenhang mit dem EPD nicht kurzfristig, sondern mittel- und langfristig beurteilen. Ein übersichtliches elektronisches Patientendossier ermöglicht es beispielsweise Ärzten, die Patienten überwiesen erhalten, sich sehr schnell einen Überblick zu verschaffen. Damit können überflüssige Zweituntersuchungen oder das aufwendige Einholen von Informationen wegfallen, um nur zwei Pluspunkte zu nennen. Ich denke, längerfristig werden mit dem EPD alle in die Behandlung Involvierten Zeit gewinnen.

Angenommen, ein Patient, der vielleicht etwas verwirrt ist, erhält eine Notfallbehandlung. Kann der behandelnde Arzt sicher sein, dass die relevanten Informationen im Dossier vorhanden und aktuell sind? Vielleicht war der Patient ja grad noch beim Spezialisten, und der hat ein neues Medikament verordnet und nicht eingetragen ...

Ich denke nicht, dass das EPD die Realität vollständig abbilden wird. Es wird so gut sein wie die vorhandenen Einträge. Es wird nicht perfekt sein, und man sollte auch keine Erwartungen an ein EPD haben, die nicht erfüllbar sind. Trotzdem wird es für die Kommunikation zwischen den verschiedenen Stellen, die mit dem Patienten zu tun haben, grosse Vorteile bieten. Die Erfahrungen aus den nordischen Ländern zeigen das deutlich.

"Ich nehme das EPD nicht als Problem, sondern als die Lösung wahr.»

Es werden sich aber möglicherweise auch Fragen rechtlicher Art stellen. Dürfen beispielsweise Einträge, die als falsch eingestuft werden, vom Arzt gelöscht werden? Wer darf das EPD «entrümpeln»? Braucht es dazu die Erlaubnis des Patienten? Wer haftet für Behandlungsfehler aufgrund falscher Einträge oder fehlender wichtiger Informationen, zum Beispiel zu Allergien? Sind solche Fragen bedacht worden? Ja, solche Fragen sind natürlich in den Vernehmlassungen zum EPD breit diskutiert worden. Viele rechtliche Fragen wie beispielsweise zur Haftung oder Stellvertre- tungen unterstehen beim EPD den gleichen Regeln wie bisher. Aber natürlich wird das EPD einen kulturellen Wandel mit sich bringen, den wir zu bewältigen haben. Wir erwähnt, wird es keinen Hauptverantwortlichen für das EPD geben - aber dafür mehr Partizipation und Eigenverantwortung des Patienten.

Wie beurteilen Sie die Auswirkungen des EPD auf die Gesundheitskosten? Dass damit Kosten gespart werden könnten, ist mittlerweile kaum noch zu hören.

«Man sollte Aufwand und Nutzen im Zusammenhang mit dem EPD mittel- und langfristig beurteilen.»

Auch im Kostenbereich wird das EPD nicht alles lösen können. Mehrfachuntersuchungen werden bestimmt reduziert werden. Das bringt neben Kosteneinsparungen eine bessere Effizienz, mehr Patientensicherheit, letztlich mehr Qualität. Wenn aber ein Arzt Mengenausweitung betreibt, wird das EPD dies nicht verhindern können. Wie die Gesamtbilanz am Schluss aussehen wird, lässt sich heute noch nicht sagen.

Was aktuell auffällt, ist eine grosse Kluft zwischen den verschiedenen "Stakeholdern". Aufseiten der "technisch" Involvierten ist fast eine Art Goldgräberstimmung zu beobachten. Auf Ärzteseite scheint die Skepsis zurzeit noch klar zu überwiegen.

Ich würde das etwas differenzierter sehen. Innerhalb der Ärzteschaft ist die Einstellung zum EPD meiner Meinung nach eine Generationenfrage. Die Motivation, ins Unterfangen EPD einzusteigen, ist im Einzelfall sehr unterschiedlich. Ich denke, die Ärztegesellschaften müssen sich nun sichtbar machen und gegenüber den Anbietern im Informatikbereich ihre Ansprüche und Sorgen kommunizieren. Seitens des BAG versuchen wir, dies zu fördern.

\section{Sie setzen für das EPD auf die junge Ärztegeneration?} Für Ärztinnen und Ärzten, die zurzeit als Assistenten im Spital arbeiten, ist der Umgang mit elektronischen Daten bereits jetzt Alltag, und sie sehen die Vorteile täglich. Dieses Wissen werden sie in die Praxis mitnehmen. Auch der Trend zu Gruppenpraxen mit Teilzeitarbeit fördert entsprechende Infrastrukturen, da es ohne Digitalisierung zusehends schwieriger wird, «vernünftig» zu arbeiten.

Sinnvoll wäre, dass die Praxissysteme mit dem EPD kommunizieren könnten und die behandlungsrelevanten Daten wie Diagnosen und Medikamente 


\section{automatisch abgeglichen würden. Wie sehen die} Perspektiven diesbezüglich aus?

Das ist genau ein Beispiel für einen der Ansprüche der Ärzteschaft, den sie von den Anbietern im Informatikbereich einfordern kann: Die möglichst reibungslose Anbindung ihrer IT-Systeme ans EPD. Dazu hat eHealth

\section{"Wie die Gesamtbilanz am Schluss aussehen wird, lässt sich heute noch nicht sagen."}

Suisse auch schon Informationsanlässe durchgeführt und Umsetzungshilfen bereitgestellt. Das Interesse vonseiten der IT-Anbieter war gross. Deshalb bin ich hier zuversichtlich.

\section{Wenn Sie einen Ausblick wagen: Wie geht es mit dem Projekt EPD nun weiter?}

Die Spitäler werden in der nächsten Phase eine wichtige Rolle spielen, weil sie eine Verpflichtung haben, das EPD in den kommenden drei Jahren auf die Beine zu stellen. Wenn sie ihren Job gut machen, wird dies dem Ganzen starken Auftrieb geben. Für mich ist zudem ganz wichtig, dass es von Anfang an keine Probleme mit der Datensicherheit gibt. Das EPD ist zu einem wesentlichen Teil auch eine Vertrauensfrage, und bei Gesundheitsdaten sind die Menschen besonders sensibel.

Bildnachweis:

Fotos Bruno Kesseli

\section{Das Elektronische Patientendossier (EPD)*}

Das Elektronische Patientendossier (EPD) ist eine Sammlung von persönlichen Dokumenten einer Patientin/ eines Patienten. Diese Dokumente enthalten behandlungsrelevante Informationen wie zum Beispiel den Austrittsbericht des Spitals, einen Pflegebericht der Spitex, Röntgenbilder, den Impfausweis oder das Rezept für die Apotheke. Patientinnen und Patienten können auch eigene Gesundheitsinfos wie die Blutdruckwerte oder ein Brillenrezept in das EPD speichern.

Um Dokumente im EPD lesen zu können, benötigen Gesundheitsfachpersonen ein explizites Zugriffsrecht. Dieses erteilt die Patientin/der Patient. Nur im medizinischen Notfall ist es möglich, ohne das Zugriffsrecht Dokumente einsehen zu können. Die Patientin/der Patient wird anschliessend über den Zugriff informiert. Organisatorisch ist das Elektronische Patientendossier ein Zusammenschluss von einzelnen autonomen Projekten. Dabei vernetzen sich Gesundheitsfachpersonen und ihre Organisationen in einem technisch-organisatorischen Verbund. Solche Verbünde heissen «EPD-Gemeinschaften».

In einigen Gemeinschaften können die Patientinnen und Patienten ihr persönliches EPD eröffnen. Solche Gemeinschaften nennt man «Stammgemeinschaften». Gemeinschaften und Stammgemeinschaften können dezentral in den Versorgungsregionen entstehen, zum Beispiel in einem Kanton oder übergreifend in mehreren Kantonen. Hier können sich alle Gesundheitsfachpersonen und ihre Einrichtungen anschliessen, also beispielsweise Spitäler, Pflegeheime, Arztpraxen, Apotheken oder Spitex-Dienste.

Umfassende Informationen zum EPD finden sich unter www.patientendossier.ch 